\title{
ON CONTINUOUS MULTIPLICATIONS ON THE TWO-SPHERE ${ }^{1}$
}

\author{
PAUL S. MOSTERT AND ALLEN L. SHIELDS
}

Since a compact manifold which admits a continuous, associative multiplication with identity must be a group [1], the two-sphere cannot be a (topological) semigroup with identity. S. T. Hu has raised the question "is it possible for the two-sphere to be a semigroup with a circle subgroup?" Here, we answer this question in the affirmative, giving both abelian and nonabelian examples. We also give some information about the structure of such semigroups which indicates that perhaps these (and similar) examples are essentially all there are. In particular, we prove the following result.

THEOREM. Let $S$ be a semigroup which is topologically the two-sphere. Suppose $B$ is a circle subgroup. Let $S \backslash B=A \cup C$, and let $e$ be the idempotent of $B$. Then

(i) $S$ has a zero, 0 , and $0 \notin B$;

(ii) If $0 \in C$, then $Q=C \cup B$ is an abelian (L)-semigroup (for the definition see \$1) with boundary $B$ and identity e. Moreover, $Q$ is contained in the center of $S$, and $Q$ is an ideal;

(iii) There exists an element $a \in A, a \in S^{2}$, such that $a x=x a=0$ for all $x \in S$.

Thinking of 0 as the south pole, $a$ as the north pole, and the northernmost circle group as the equator, this gives complete information about the southern hemisphere, and a hint as to what happens in the northern. Probably, there is a northern "cap" which multiplies by projection onto the southern hemisphere, with the identity of the southern hemisphere acting as the projector. The cylinder between the southern hemisphere and the northern cap is probably of the form $J \times B$ where $J$ is a semigroup on the unit interval whose multiplication "collapses" in some manner (see our examples) to its only idempotent which is a zero at one endpoint. We have been unable to recover this cylinder (if it exists).

Presented to the Society, November 19, 1955; received by the editors August 30, 1955.

1 This work was done during the summer of 1955 while the authors were at the Institute for Advanced Study. They received their support from Tulane's National Science Foundation contract. 
1. Preliminaries. By semigroup we shall always mean topological semigroup (i.e., a Hausdorff space with a continuous, associative multiplication). A subsemigroup will always be closed. An ideal of $S$ is a subset $R \subset S$ such that $x \in R, y \in S$ implies $x y \in R, y x \in R$. An ideal may or may not be closed. The kernel $K$ of $S$ is the (unique) minimal closed ideal of $S$. We use $\square$ to denote the null set. $A \backslash B$ is used in place of $A-B$.

We recall here that an (L)-semigroup is a semigroup with identity on a compact manifold with connected boundary such that the boundary is a (Lie) subgroup. The authors [2] have completely classified all (L)-semigroups. Of particular use here is the following result which is a special case of the more general problem treated in [2]:

1.1. Lemma. Let $Q$ be a two-cell which is an (L)-semigroup. Then $Q$ is abelian. In fact, if $B$ is the boundary of $Q$, then $B$ is a circle group, and is the largest group containing the identity. Further, there is a subsemigroup $J \subset Q$ such that $J$ is isomorphic and homeomorphic to a semigroup on $[0,1]$ with 0 acting as a zero, and 1 as an identity. Moreover, the zero for $J$ is a zero for $Q$, and $J B=Q$.

The authors [2] have also classified semigroups on $[0,1]$ of the type described above, but this information is used only indirectly here, i.e., only insofar as it helps describe the (L)-semigroup $Q$.

1.2. Lemma. Let $D$ be the two-cell (unit disc) in the complex plane, $|z| \leqq 1$, and $U=\left\{z:|z|<r_{0} \leqq 1\right\}$. If the mapping $f: D \rightarrow D$ is such that $f(z)=z$ for $|z|=r_{0}$, then $U \subset f(U)$.

Proof. This is quite easy using the index of the mapping.

1.3. Lemma. Let $D$ be the two-cell, $D=\mathrm{U}_{a} C_{a}$ where $C_{a}$ is a circle, and $C_{a} \cap C_{b}=\square$ if $a \neq b$. Then at least one $C_{a}$ is degenerate (i.e., a point).

Proof. Let $D_{a}$ equal $C_{a}$ together with its interior, and partially order the sets $D_{a}$ by inclusion. Let $\mathcal{F}$ be a maximal chain, and $P=\bigcap_{a} D_{a}$ for $D_{a} \in \mathcal{F}$. Then $P$ is nonempty since each $D_{a}$ is compact. Let $x \in P$, and suppose $x \in C_{b}$. Clearly $D_{b} \subset D_{a}$ for every $D_{a} \in \mathcal{F}$, and therefore, since $\mathcal{F}$ is maximal, $D_{b} \in \mathcal{F}$ and $D_{b}$ is a minimal element. Hence there can be no $c \neq b$ such that $D_{c} \subset D_{b}$. But this implies $C_{b}=D_{b}=\{x\}$.

2. Proof of the theorem. We shall assume, for the remainder of this section, that $S$ is the two-sphere; that $S$ is a semigroup; that $S$ contains a circle subgroup $B$ with idempotent (identity for $B$ ) $e$; and that $S \backslash B=A \cup C$. Here $A$ and $C$ are disjoint, open hemispheres, each homeomorphic with an open two-cell, and the boundary of each is $B$. 
The proof proceeds in a number of short steps.

2.1. Define $f: S \rightarrow S$ by $f(x)=x e$. If $A \nsubseteq f(S)$, then $C \subset f(S)$.

Proof. Since $A$ is not contained in $f(S)$, there is a small open disc $W \subset A, W \cap f(S)=\square$. Let $V=S \backslash W$. Then $V$ is a closed two-cell. Further, $f(V) \subset V$ (since $f(S) \subset V$ ) and $f(b)=b$ for $b$ in $B$. By Lemma 1.2, $C \subset f(C) \subset f(S)$.

Assume hereafter, then, that $C \subset f(S)$.

2.2. For $x \in Q=C \cup B, x e=x$.

Proof. Since $C \subset f(S)$, each $x \in C$ is of the form ye for some $y \in S$. Hence, $x=y e=(y e) e=x e$.

2.3. If $K$ is the minimal ideal of $S$, then $B \cap K=\square$.

Proof. If $B$ meets $K$, then clearly $B \subset K$. But $K$ is the union of disjoint, connected, maximal subgroups topologically isomorphic to one another [1]. Let $R$ be the maximal subgroup containing $e$. We then have $R=B$. For if not, since $R$ is connected, we would have $\operatorname{dim} R>1$ [3] so that $\operatorname{dim} R=2$. Hence, $R$ contains an open neighborhood of $e$ (it must contain a two-dimensional Euclidean subset), and hence $R$ is a 2-dimensional compact Lie group. But a compact manifold does not contain a proper compact submanifold of the same dimension [4, see proof of 3.1]. Hence, $R=S$ which is impossible.

Since $e \in K, f(S) \subset K$, where $f$ is the mapping of (2.1), and in particular $C \subset f(S) \subset K$. It follows that $C$ is a union of disjoint, nondegenerate, circle groups. But this contradicts Lemma 1.3.

2.4. $Q=C \cup B$ is an ideal.

Proof. Let $K$ denote the unique minimal ideal of $S$. For any set $R \subset S$, let $J(R)$ denote the union of all ideals (not necessarily closed) contained in $R$, if any such exist. Then $J(R)$ is an ideal, the largest ideal contained in $R$. It is known [5] that if $R$ is open and contains at least one ideal, then $J(R)$ is open.

$K$ is connected $(K=S k S$ for any $k \in K$ ), so by 2.3 either $K \subset A$ or $K \subset C$. Assume $K \subset A$. Then $J(A)$ is open. $J(A)^{-}$is an ideal bigger than $J(A)$, so it meets $B$. Hence $B \subset J(A)^{-}$, and in particular, $e \in J(A)^{-}$. But by 2.2, $C=C e \subset J(A)^{-} \subset A \cup B$ which is clearly impossible.

Thus, $K \subset C$, and therefore $J(C)$ is open. Then, just as above, $B C J(C)^{-}$, and therefore $C \subset J(C)^{-}$. But this means $J(C)^{-}=Q$.

2.5. $Q$ is an abelian (L)-semigroup with identity e, and zero 0 .

Proof. Topologically $Q$ is a two-cell with boundary $B$ and, by 2.5 , $Q$ is a subsemigroup. From 2.2 we know that $e$ is a right identity for $Q$. Consider now the function $g(x)=e x$. It maps $Q$ into itself, and leaves $B$ pointwise fixed, and hence is onto (Lemma 1.2). As in 2.2 , this implies that $e x=x$ for each $x \in Q$, so $e$ is also a left identity 
for $Q$. Thus $Q$ is a two-cell (L)-semigroup with identity $e$ and boundary $B$. The rest of the assertion now follows from Lemma 1.1.

2.6. S has a zero.

Proof. Let 0 be the zero of $Q$. Since $Q$ is an ideal, $0 x \in Q$ for all $x \in S$. Hence $0 x=0^{2} x=0(0 x)=0$. Similarly, $x 0=0$.

2.7. $Q$ is in the center of $S$.

Proof. Let $q \in Q, x \in S$, so that $q x \in Q$ and hence $q x=e q x e, q=e q$. Then since $Q$ is abelian:

$$
q x=(e q)(x e)=(x e)(e q)=x(e e q)=x(e q)=x q .
$$

2.8. If $f: A \cup B \rightarrow C \cup B$ is a mapping such that $f(b)=b$ for $b \in B$, then $f$ is onto.

Proof. Let $p$ be a 1-1 continuous projection of $A \cup B$ onto $C \cup B$ leaving $B$ pointwise fixed. Then $F: C \cup B \rightarrow C \cup B$ defined by $F(x)$ $=f\left(p^{-1}(x)\right)$ leaves $B$ pointwise fixed, and hence is onto (Lemma 2.1). This clearly implies the result.

2.9. If $x y \in A \cup B$, then $e x, e y \in B$.

Proof. Since $x S$ is connected and meets both $A \cup B$ and $Q$, it meets $B$. It follows that there is a point $z \in S$ such that $x z=e$. Hence, $e=(e x)(e z)$ so that $e z$ is an inverse for $e x$. Since $e x \in Q$, this implies $e x \in B$. Similarly, $e y \in B$.

We now complete the proof of (iii) of the theorem. By (2.8) the mapping defined by $f(x)=x e$ is onto, so that there exists an element $a \in A$ such that $e a=a e=0$. Now $a S \cup S a \subset Q$, for if not (say $a S \nsubseteq Q$ ), there exists some $x \in S$ such that $a x \in A$. By (2.9) this implies $a e \in B$, a contradiction. Hence for $x \in S, a x \in Q$. But $e$ is the identity for $Q$ so that $a x=e a x=0 x=0$. Similarly, $x a=0$ for all $x$. Now clearly $a \notin S^{2}$, for if $x y=a$, then by (2.9), $x e, y e \in B$. Hence, $0=a e=x y e=(x e)(y e)$ $\in B$, a contradiction. This completes the proof.

3. Some examples. We give here two examples (many more could be constructed similarly) of semigroups on the two-sphere having circle subgroups. In the process we give an interesting example of a nonabelian semigroup on a closed interval, (Example 3.2). It is known [6] that if a closed interval is a semigroup with one end point a zero and the other an idempotent, then it must be abelian. Example 3.2 shows this cannot be relaxed to just the assumption that one endpoint is a zero.

3.1. Example. Let $I_{1}$ be the unit interval $[0,1]$, and define $x y=0$ for all $x, y \in I$.

3.1'. ExAmple. Let $D$ be any (L)-semigroup on a 2-cell with boundary $B$. Let $P=B \times I_{1}$, and let $D_{0}$ be another copy of $D$. Paste $D, P$, and $D_{0}$ together in the obvious way so that the base $(=B \times\{0\})$ 
of $P$ coincides with $B$ of $D$, and the top $(=B \times\{1\})$ coincides with $B_{0}$ of $D_{0}$. We thus obtain a two-sphere. Define multiplication in $D$ and in $P$ in the natural way (they are already semigroups). To multiply an element of $P$ by an element of $D$, first project the element of $P$ into $B$, and then take the multiplication for elements of $D$. Finally, to multiply elements in $D_{0}$ by anything, we first project them into the corresponding elements in $D$, and then use the previously defined multiplication. The multiplication so defined is continuous and associative, and $B$ is a circle subgroup (in fact, depending on our choice of $D$, we may have a continuum of circle subgroups, [2]). Note that this semigroup is abelian.

3.2. ExAmple. Let $I_{2}$ be the interval $[1 / 8,1 / 2]$ and let $f:[1 / 3$, $1 / 2] \rightarrow[1 / 8,1 / 3]$ be any homeomorphism such that $f(1 / 3)=1 / 3$ and $f(1 / 2)=1 / 8$. Denote the ordinary product of $x$ and $y$ by $x y$, and define a multiplication in $I_{2}$, to be denoted $x \circ y$, as follows:

(i) $x \in[1 / 8,1 / 3], \quad y \in I_{2}$, then $x \circ y=\max (1 / 8, x y)$;

(ii) $x \in[1 / 3,1 / 2], \quad y \in I_{2}$, then $x \circ y=f(x) \circ y$.

Since all triple products are zero $(=1 / 8)$, the associativity of this multiplication is trivial. Continuity is clear by the definition. Since $(1 / 2) \circ(1 / 3)=1 / 8$, and $(1 / 3) \circ(1 / 2)=1 / 6, I_{2}$ is not abelian.

Now take $I_{2}^{\prime}=[0,1]$ such that $[0,1 / 2]$ is isomorphic to $I_{2}$ above, and multiplication $x \circ y$ is defined for elements $x \in[0,1 / 2], y \in[1 / 2$, 1] by:

$$
x \circ y=x \circ(1-y), \quad y \circ x=(1-y) \circ x
$$

and for $x, y \in[1 / 2,1]$ by:

$$
x \circ y=(1-x) \circ(1-y) \text {. }
$$

3.2'. Example. Construct a semigroup on the two-sphere as in $3.1^{\prime}$, with $I_{2}^{\prime}$ taking the place of $I_{1}$. This example is nonabelian.

It would be interesting to know whether all semigroups on the twosphere (of the type considered here) are obtained in like manner.

4. Some problems. This question leads to some interesting problems about the two-sphere. We list here three that are of interest to us.

P1. Is every nonzero idempotent to the sphere contained in a circle subgroup, if there exists at least one such?

P2. If $B_{0}$ is the circle group nearest $a$ (see the theorem), is the (L)-semigroup $D_{0}$ consisting of the hemisphere below (and including) $B_{0}$ the maximal ideal $J$ with the property that $J^{2}=J$ ? 
P3. We know that under our assumptions, $S^{2} \neq S$. If the two. sphere is a semigroup $S$ such that $S^{2}=S$, must the multiplication be trivial (i.e., either $x y=x$ for all pairs, or $x y=y$ always)? This is a special case of a problem of A. D. Wallace [7], namely, if $M$ is a compact connected manifold which is a semigroup such that $M^{2}=M$, must one of the following hold: (i) $M$ is a group, or (ii) multiplication is trivial.

This is in general false. For example, consider the torus $T=A \times B$, where $A$ and $B$ are circles. Give $A$ the circle group multiplication, and $B$ the trivial multiplication: $b c=c$ for all $b, c \in B$. Give $T$ the direct product multiplication. Then $T^{2}=T$, but neither (i) nor (ii) holds.

\section{BIBLIOGRAPHY}

1. A. D. Wallace, The structure of topological semigroups, Bull. Amer. Math. Soc. vol. 61 (1955) pp. 95-112.

2. P. S. Mostert and A. L. Shields, On the structure of semigroups on a compact manifold with boundary, to appear in Ann. of Math.

3. L. Pontrjagin, Topological groups, Princeton, 1939.

4. H. C. Wang, Closed manifolds with homogeneous complex structure, Amer. J. Math. vol. 76 (1954) pp. 1-32.

5. R. J. Koch and A. D. Wallace, Maximum ideals in compact semigroups, Duke Math. J. vol. 21 (1954) pp. 681-686.

6. W. M. Faucett, Compact semigroups irreducibly connected between two idempotents, Proc. Amer. Math. Soc. vol. 6 (1955) pp. 741-747.

7. A. D. Wallace, Manifolds with multiplication, Bull. Amer. Math. Soc. Research Problem 61-1-7.

The Tulane University of Louisiana 